\title{
Higgs-Yukawa model on the lattice ${ }^{\star}$
}

\author{
David Y.-J. Chu ${ }^{1}$, Karl Jansen ${ }^{2}$, Bastian Knippschild ${ }^{3}$, and C.-J. David Lin ${ }^{4, \star \star ~}$ \\ ${ }^{1}$ Department of Electrophysics, National Chiao-Tung University, Hsinchu 30010, Taiwan \\ ${ }^{2}$ NIC, DESY Zeuthen, D-15738, Germany \\ ${ }^{3}$ HISKP and Bethe Centre for Theoretical Physics, University of Bonn, D-53115, Germany \\ ${ }^{4}$ Institute of Physics, National Chiao-Tung University, Hsinchu 30010, Taiwan
}

\begin{abstract}
We present results from two projects on lattice calculations for the HiggsYukawa model. First we report progress on the search of first-order thermal phase transitions in the presence of a dimension-six operator, with the choices of bare couplings that lead to viable phenomenological predictions. In this project the simulations are performed using overlap fermions. Secondly, our study for applying finite-size scaling techniques near the Gaussian fixed point of the Higgs-Yukawa model is presented. We discuss the analytical formulae for the Higgs Yukawa model and show results for a first numerical study in the pure $O(4)$ scalar sector of the theory.
\end{abstract}

\section{Introduction}

Investigation of the Higgs-Yukawa model using Lattice Field Theory can result in useful input for physics at the Large Hadron Collider (LHC). In particular, it enables us to extend the study of the model to the non-perturbative regime where a rich phase structure is being found [1-4]. Such a phase structure can be employed to address the hierarchy problem and the issue of triviality. It can also provide insight into the nature of electroweak thermal phase transition which plays an important role in phenomenology of baryogenesis.

This article presents two projects on the Higgs-Yukawa model. First we discuss the extension of the theory with a dimension-six operator that may lead to a strong first-order thermal phase transition [5-9]. Secondly, we show results from our study of finite-size scaling for the Higgs-Yukawa model near the Gaussian fixed point. Such a study will allow us to develop useful tools in looking for possible non-trivial fixed points at strong coupling.

In this work, we investigate the Higgs-Yukawa model that is described by the continuum action

$$
\begin{aligned}
S^{\text {cont }}[\varphi, \bar{\psi}, \psi]= & \int \mathrm{d}^{4} x\left\{\frac{1}{2}\left(\partial_{\mu} \varphi\right)^{\dagger}\left(\partial_{\mu} \varphi\right)+\frac{1}{2} m_{0}^{2} \varphi^{\dagger} \varphi+\lambda\left(\varphi^{\dagger} \varphi\right)^{2}\right\} \\
& +\int \mathrm{d}^{4} x\left\{\bar{\Psi} \not \partial \Psi+y\left(\bar{\Psi}_{L} \varphi b_{R}+\bar{\Psi}_{L} \tilde{\varphi} t_{R}+\text { h.c. }\right)\right\}, \\
& \text { where } \varphi=\left(\begin{array}{c}
\phi_{2}+i \phi_{1} \\
\phi_{0}-i \phi_{3}
\end{array}\right), \quad \tilde{\varphi}=i \tau_{2} \varphi, \quad \Psi=\left(\begin{array}{c}
t \\
b
\end{array}\right), \quad \Psi_{L, R}=\frac{1 \mp \gamma_{5}}{2} \Psi,
\end{aligned}
$$

${ }^{\star}$ Preprint number DESY $17-164$

${ }^{\star \star}$ Speaker, e-mail: dlin@mail.nctu.edu.tw 
with $\phi_{i}$ being real scalar fields, $t$ and $b$ being the "top" and the "bottom" quark fields, and $\tau_{2}$ being the second Pauli matrix. Amongst the scalar fields, the component $\phi_{0}$ will develop a non-vanishing vacuum expectation value (vev) in the phase of spontaneously-broken $O(4)$ symmetry. The above action contains three bare couplings, $m_{0}, \lambda$ and $y$. Notice that we employ degenerate Yukawa couplings in this work. To discretise this action, we resort to overlap fermions that allow us to properly define the lattice version of the left- and right-handed fermions in the Yukawa terms. Furthermore, we follow the convention in representing the bosonic component of the lattice action as

$$
S_{B}[\Phi]=-\kappa \sum_{x, \mu} \Phi_{x}^{\dagger}\left[\Phi_{x+\mu}+\Phi_{x-\mu}\right]+\sum_{x} \Phi_{x}^{\dagger} \Phi_{x}+\hat{\lambda} \sum_{x}\left[\Phi_{x}^{\dagger} \Phi_{x}-1\right]^{2}
$$

where $\kappa$ is the hopping parameter, $x$ labels lattice sites, and $\mu$ specifies the space-time directions. The relationship between the lattice and the continuum bosonic fields and relevant couplings is

$$
a \varphi=\sqrt{2 \kappa} \Phi_{x} \equiv \sqrt{2 \kappa}\left(\begin{array}{c}
\Phi_{x}^{2}+i \Phi_{x}^{1} \\
\Phi_{x}^{0}-i \Phi_{x}^{3}
\end{array}\right), \quad \lambda=\frac{\hat{\lambda}}{4 \kappa^{2}}, \quad m_{0}^{2}=\frac{1-2 \hat{\lambda}-8 \kappa}{\kappa}
$$

where $a$ denotes the lattice spacing.

All the numerical works reported in this article have been performed with the choice of the bare Yukawa coupling,

$$
y=175 / 246
$$

as motivated by the physical values of the Higgs-field vev and the top-quark mass.

\section{Thermal phase transition in the Higgs-Yukawa model with a dimension-six operator}

In this section, we present our study of the Higgs-Yukawa model with the inclusion of a term,

$$
O_{6}=\frac{\lambda_{6}}{\Lambda^{2}} \int \mathrm{d}^{4} x\left(\varphi^{\dagger} \varphi\right)^{3}
$$

in the action of Eq. (1). This term can serve as a prototype of new physics. Here $\Lambda$ is the cut-off scale that can be realised as $1 / a$ on the lattice. It is natural to include this operator as one of the higher-dimension terms when interpreting the Higgs-Yukawa model in the language of effective field theory [10]. In the above expression, $\lambda_{6}$ is dimensionless. The addition of the dimension-six operator, $\left(\varphi^{\dagger} \varphi\right)^{3}$, can enrich both thermal and non-thermal phase structures of the theory.

Our main interest in the Higgs-Yukawa model with the operator in Eq. (5) is the search for a viable scenario for a strong first-order phase transition in the theory. In performing such search, we scan the bare-coupling space to identify choices of parameters such that

1. The cut-off is high enough compared to the Higgs-field vev, denoted as $\langle\varphi\rangle \equiv\left\langle\phi_{0}\right\rangle$. This means the condition,

$$
a\langle\varphi\rangle \ll 1,
$$

has to be satisfied in our simulations.

2. The ratio between $\langle\varphi\rangle$ and the Higgs-boson mass is compatible with experimental results, i.e.,

$$
\frac{\langle\varphi\rangle}{m_{H}} \sim 2
$$


3. The thermal phase transition is first-order.

To ensure that Eq. (6) is realised in our simulations, we have to examine the non-thermal phase structure of the model. In the phase where the $O(4)$ symmetry is spontaneously broken, this condition can be satisfied near any second-oder non-thermal phase transitions. In our previous work [11], a thorough investigation in this regard was conducted for two choices of $\lambda_{6}\left(\lambda_{6}=0.001\right.$ and $\left.\lambda_{6}=0.1\right)$, leading to useful information for the current study. In order to check the constraint of Eq. (7), we determine the Higgs-boson mass from the momentum-space Higgs propagator.

An important tool in our study of the phase structures is the constraint effective potential (CEP) $[12,13]^{1}$. The CEP, $U(\hat{v})$, is a function of the Higgs-field zero mode,

$$
\hat{v}=\frac{1}{V}\left|\sum_{x} \Phi_{x}^{0}\right|,
$$

where $V$ is the four-volume and the sum is over all lattice points. This effective potential can be calculated analytically using perturbation theory. It can also be extracted numerically through a histogramming procedure of $\hat{v}$ in Monte-Carlo simulations.

Figure 1 exhibits our results at $\lambda_{6}=0.001$ and $\lambda=-0.008$. According to the study in Ref. [11], perturbative calculations of the CEP are reliable at these values of the couplings. The plot in the left-hand panel demonstrates that this choice of the self couplings can lead to a second-order nonthermal and a first-order thermal phase transitions. The first-order transition is further evidenced by our numerical study of the CEP, as presented in the right-hand panel. On the other hand, results from perturbation theory show that the ratio $\langle\varphi\rangle / m_{H}$ does not satisfy the condition in Eq. (7).
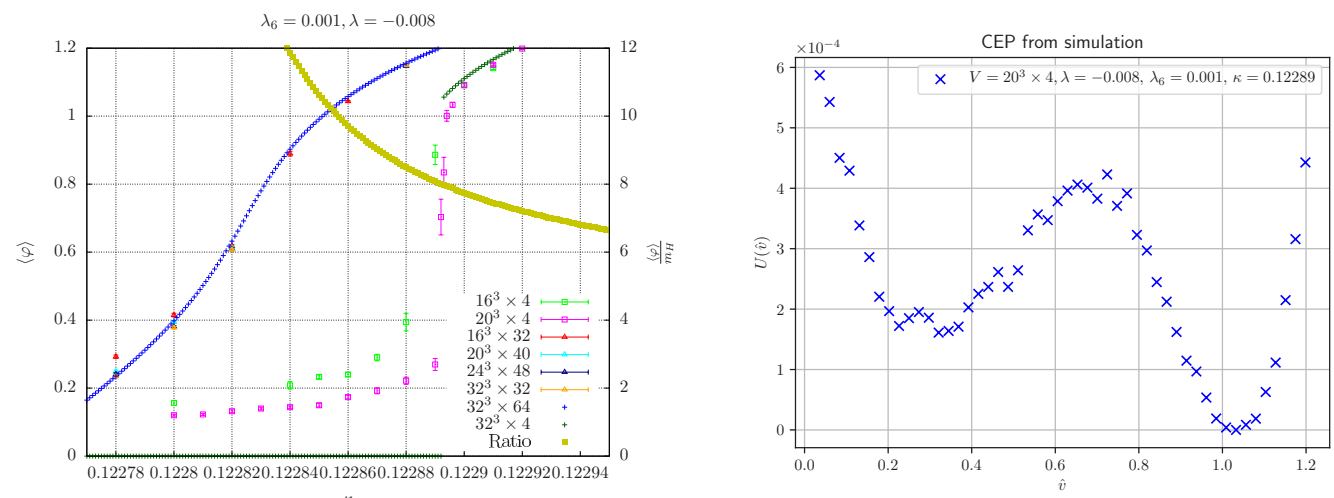

Figure 1. Results for $\lambda_{6}=0.001$ and $\lambda=-0.008$. In the left-hand panel, the errors are statistical from lattice computations, and symbols without errors represent results obtained in perturbative calculations using the constraint effective potential. "Ratio" means $\langle\varphi\rangle / m_{H}$. In this figure, the Higgs vev, $\langle\varphi\rangle$, is plotted in lattice units. The right-hand side is the constraint effective potential, obtained in the Monte-Carlo simulation, at the four-volume $(L / a)^{3} \times(T / a)=20^{3} \times 4$ and $\kappa=0.12289$.

To perform further search, we choose $\lambda_{6}=0.1$ and $\lambda=-0.378$. Our work in Ref. [11] shows that perturbation theory for the CEP is no longer reliable at this value of $\lambda_{6}$. Therefore we only resort to Monte-Carlo simulations on the lattice. Results for the CEP near the phase transitions are displayed in Fig. 2. Although it is not clear whether these transitions are first- or second-order, it can be concluded

\footnotetext{
${ }^{1}$ An alternative, somewhat similar, tool for such studies is the extended mean-field theory [14].
} 

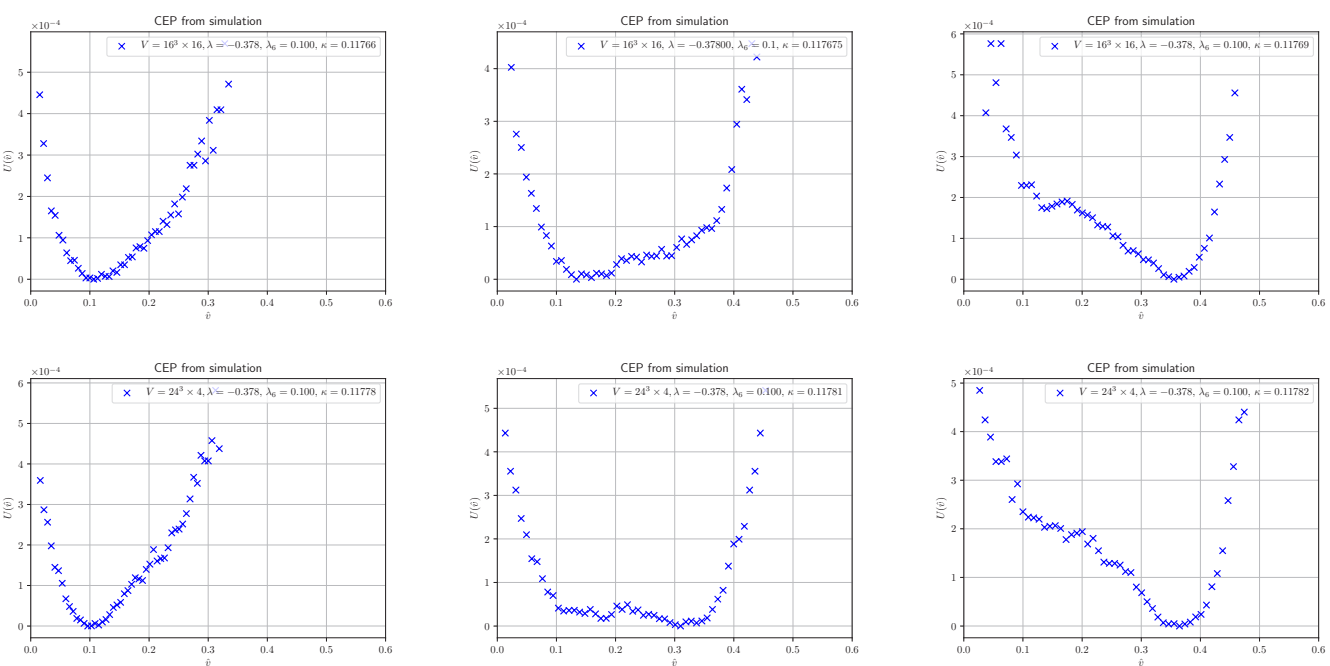

Figure 2. Results for $\lambda_{6}=0.1$ and $\lambda=-0.378$. The plots on the first row are the CEP at three representative values of $\kappa$ near the non-thermal phase transition. The second row displays their counterparts at the finitetemperature transition.

that the non-thermal and the thermal phase transitions exhibit almost the same properties. Hence we conclude that this choice of the bare scalar self-couplings does not lead to a viable scenario for a strong first-order thermal phase transition.

Presently we are continuing this search with other choices of $\lambda_{6}$ and $\lambda$.

\section{Finite-size scaling for the Higgs-Yukawa model near the Gaussian fixed point}

In this project, we study the Higgs-Yukawa model as described by the continuum action in Eq. (1), i.e, we have

$$
\lambda_{6}=0,
$$

and only include operators with dimension less than or equal to four in the action. The purpose of this investigation is to develop tools for exploring the scaling behaviour of the model near the Gaussian fixed point. These tools can be used to confirm the triviality of the Higgs-Yukawa theory, or to search for alternative scenarios where strong-coupling fixed points exist. Predictions from perturbation theory indicate the possible appearance of non-trivial fixed points in the Higgs-Yukawa model [15]. This issue was also examined with the approach of functional renormalisation group [16], where no nonGaussian fixed point was found. Nevertheless, early lattice computations showed evidence for the opposite conclusion [1]. We stress that an extensive, non-perturbative study of the Higgs-Yukawa theory from first-principle calculations is still wanting. This is in contrast to the situation of the purescalar models which are now widely believed to be trivial (see, e.g., Refs. [17, 18]).

In our previous attempt at addressing the issue of the triviality in the Higgs-Yukawa model, as reported in Ref. [4], we employed the technique of finite-size scaling. The main finding in Ref. [4] is that one needs to understand the logarithmic corrections to the mean-field scaling behaviour, in order 
to draw concrete conclusions. In view of this, we developed a strategy, and worked out its analytic aspects, as reported in Refs. [19, 20].

The main result in Refs. [19, 20] is that, using the techniques established by Zinn-Justin and Brezin for scalar field theories [21], we can derive finite-size scaling formulae for various quantities in one-loop perturbation theory near the Gaussian fixed point. It is natural to include the leadingorder logarithmic corrections to the mean-field scaling law through this procedure. In this strategy, we first match correlators obtained with lattice regularisation to an on-shell renormalisation scheme, with the matching scale chosen to be the pole mass, $m_{P}$, of the scalar particle. This pole mass can be extracted by studying the scalar-field propagator on the lattice. Its relationship with the renormalised mass parameter [the renormalised counterpart of the bare coupling $m_{0}$ in Eq. (1)], $m$, in the theory is

$$
\begin{aligned}
& m^{2}\left(m_{P}\right)=m_{P}^{2} \text { in the symmetric phase, } \\
& m^{2}\left(m_{P}\right)=-\frac{1}{2} m_{P}^{2} \text { in the broken phase, }
\end{aligned}
$$

where the renormalisation scale is $m_{P}$. Notice that $m_{P}$ is the Higgs-boson pole mass in the broken phase. Under the assumption that we work closely enough to the critical surface of the Gaussian fixed point, the condition $m_{P} \ll 1 / a$ is satisfied. We can then carry out one-loop running of the renormalised correlators from $m_{P}$ to another low-energy scale that is identified with the inverse lattice size, $L^{-1}$, that is of the same order of $m_{P}$ but with the constraint $m_{P} L>1$. This leads to predictions of finite-size scaling behaviour of these correlators. In performing the above one-loop running, one has to solve the relevant renormalisation group equations, introducing integration constants. These constants will then be treated as fit parameters when confronting the scaling formulae with lattice numerical data.

Up to the effect of wavefunction renormalisation, which results in additional $L$-dependence that can also be accounted for using one-loop perturbation theory, we have found that all the correlators containing only the zero mode of the scalar field can be expressed in terms of a class of functions

$$
\begin{aligned}
& \bar{\varphi}_{0}(z)=\frac{\pi}{8} \exp \left(\frac{z^{2}}{32}\right) \sqrt{|z|}\left[I_{-1 / 4}\left(\frac{z^{2}}{32}\right)-\operatorname{Sgn}(z) I_{1 / 4}\left(\frac{z^{2}}{32}\right)\right], \\
& \bar{\varphi}_{1}(z)=\frac{\sqrt{\pi}}{8} \exp \left(\frac{z^{2}}{16}\right)\left[1-\operatorname{Sgn}(z) \operatorname{Erf}\left(\frac{|z|}{4}\right)\right], \bar{\varphi}_{n+2}(z)=-2 \frac{\mathrm{d}}{\mathrm{d} z} \bar{\varphi}_{n}(z),
\end{aligned}
$$

where the scaling variable, $z$, is

$$
z=\sqrt{s} m^{2}\left(L^{-1}\right) L^{2} \lambda_{R}\left(L^{-1}\right)^{-1 / 2},
$$

with $\lambda_{R}\left(L^{-1}\right)$ being the quartic coupling renormalised at the scale $L^{-1}$, and $s$ being the anisotropy of the four-volume, $L^{3} \times T=L^{3} \times s L$. Notice that the $L$-dependence in $\lambda_{R}\left(L^{-1}\right)$ can be complicated, involving two integration constants resulted from solving the renormalisation group equations for the Yukawa and the quartic couplings [22]. On the other hand, there is no unknown parameter in the renormalised mass, $m\left(L^{-1}\right)$, because it is obtained from the scalar pole mass computed numerically on the lattice.

In this work, we study the Higgs-field vev, $\langle\varphi\rangle \equiv\left\langle\phi_{0}\right\rangle$, its susceptibility, $\chi$, and Binder's cumulant, $Q$. The finite-size scaling formulae for these quantities are found to be

$$
\begin{aligned}
\langle\varphi\rangle & =s^{-1 / 4} A^{(\varphi)} L^{-1}\left[\lambda_{R}\left(L^{-1}\right)\right]^{-1 / 4} \frac{\bar{\varphi}_{4}(z)}{\bar{\varphi}_{3}(z)} \\
\chi & =s L^{4}\left(\left\langle\varphi^{2}\right\rangle-\langle\varphi\rangle^{2}\right)=s^{1 / 2} A^{(\chi)} L^{2}\left[\lambda_{R}\left(L^{-1}\right)\right]^{-1 / 2}\left[\frac{\bar{\varphi}_{5}(z)}{\bar{\varphi}_{3}(z)}-\left(\frac{\bar{\varphi}_{4}(z)}{\bar{\varphi}_{3}(z)}\right)^{2}\right], \\
Q & =1-\frac{\left\langle\varphi^{4}\right\rangle}{3\left\langle\varphi^{2}\right\rangle^{2}}=1-\frac{\bar{\varphi}_{7}(z) \bar{\varphi}_{3}(z)}{3 \bar{\varphi}_{5}(z)^{2}}
\end{aligned}
$$


where $\left\langle\varphi^{2}\right\rangle \equiv\left\langle\phi_{0}^{2}\right\rangle$ in the definition of the susceptibility, $A^{(\varphi)}$ and $A^{(\chi)}$ are unknown constants resulting from integrating the renormalisation group equation for the the wavefunction.

As mentioned above, the formulae in Eq. (13) can be complicated in the Higgs-Yukawa theory. Therefore as the first numerical test of our strategy, we resort to the pure-scalar $O(4)$ model, in which the one-loop $\lambda_{R}\left(L^{-1}\right)$ takes the simple form,

$$
\lambda_{R}\left(L^{-1}\right)=\frac{\lambda_{m_{P}}}{1+\frac{6}{\pi^{2}} \log \left(m_{P} L\right)},
$$

with only one integration constant, $\lambda_{m_{P}} \equiv \lambda_{R}\left(m_{P}\right)$, to be fitted from numerical data. In this numerical test, the bare quartic coupling is chosen to be 0.15 , to ensure that we are working in the perturbative regime. Throughout our analysis procedure, the scalar-particle pole mass is determined by fitting the four-momentum space propagator, and then extrapolated to the infinite-volume limit employing a ChPT-inspired formula. More details of this aspect of our work will be reported in a future publication [22].

Figure 3 shows the results of the fits for $\langle\varphi\rangle, \chi$ and $Q$ in the pure-scalar $O(4)$ model using the scaling formulae in Eqs. (13) and (14). The fit parameters are $\lambda_{m_{R}}$ and $A^{(\varphi, \chi)}$. We distinguish them in the symmetric and the broken phases, since the numerical values of these parameters need not be the same in two different phases. Upon extracting these parameters from our lattice numerical result, we can then evaluate the scaling variable, $z$, for our data points, as well as removing (rescaling away) the volume-dependence introduced via the effect of the wavefunction renormalisation in Eq. (13). This allows us to test our finite-size scaling formulae through plotting the rescaled $\langle\varphi\rangle, \chi$ and $Q$ as functions of $z$. In Fig. 4, it is demonstrated that these rescaled quantities all lie on universal curves that are only functions of $z$. Such behaviour, together with the good or reasonable values of the $\chi^{2}$, show strong evidence that our formulae indeed capture the scaling properties of the theory as governed by the Gaussian fixed point.
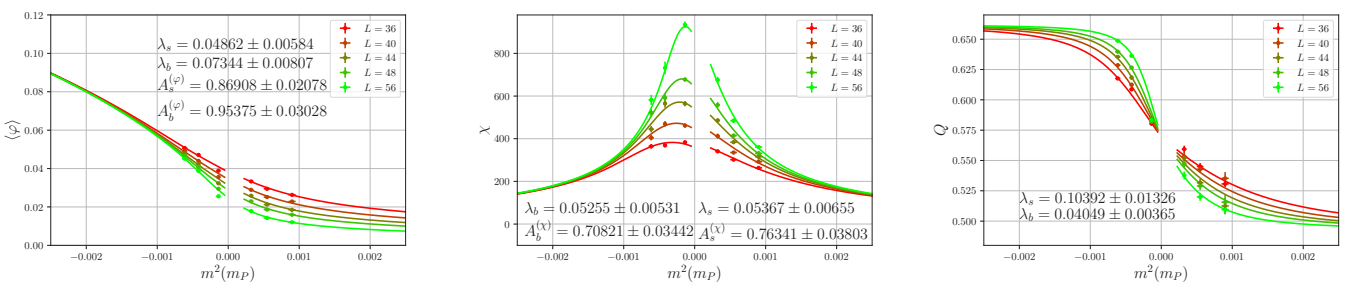

Figure 3. Results for the fit of the Higgs-field vev, its susceptibility and Binder's cumulant using the finite-size scaling formulae in the pure-scalar $O(4)$ model. The parameters $\lambda_{s, b}$ are $\lambda_{m_{R}}$ in the symmetric and the broken phases, with similar symbols indicating the relevant $A^{(\varphi)}$ and $A^{(\chi)}$ in these two phases. All dimensionfull quantities are expressed in lattice units.

\section{Conclusion and outlook}

In this article, we present two projects on lattice simulations for the Higgs-Yukawa model. The results from our study show that Lattice Field Theory can be employed for investigating non-perturbative aspects of the model.

Our first project is the search for phenomenologically viable scenarios for a strong first-order thermal phase transition in the Higgs-Yukawa theory with the addition of a dimension-six operator. 

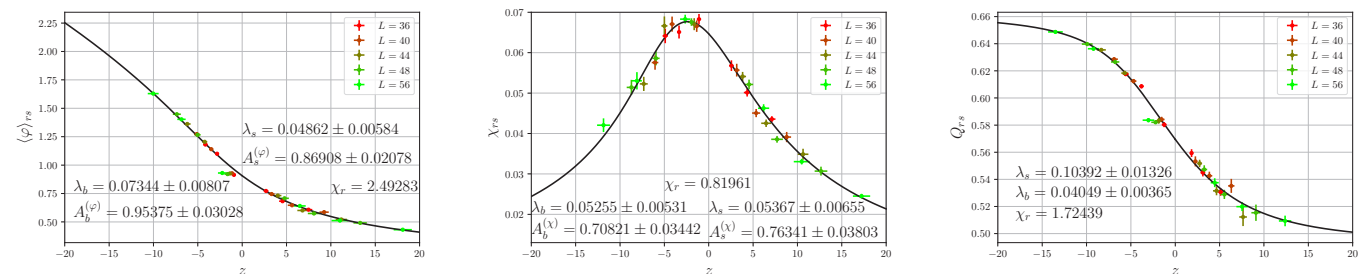

Figure 4. Scaling behaviour of the Higgs-field vev, its susceptibility and Binder's cumulant in the pure-scalar $O(4)$ model. All dimensionful quantities are expressed in lattice units. The subscript $r s$ means these quantities are rescaled properly to remove the volume dependence introduced via the effect of wavefunction renormalisation. The parameters $\lambda_{s, b}$ are $\lambda_{m_{R}}$ in the symmetric and the broken phases, with similar symbols indicating the relevant $A^{(\varphi)}$ and $A^{(\chi)}$ in these two phases, and $\chi_{r}$ indictes the $\chi^{2} /$ d.o.f. of the fit.

This dimension-six operator can serve as a prototype of new physics. In this work, we demonstrate that such first-order transitions can indeed be observed, when the cut-off scale is kept high in comparison to the Higgs-boson vev. However, we are yet to find a suitable choice of parameters which leads to large enough Higgs-boson mass. Currently we are performing more lattice computations to further scan the bare parameter space.

The second project presented in this article is our work on finite-size scaling behaviour of the Higgs-Yukawa model near the Gaussian fixed point. In this regard, we have derived the scaling formulae by solving the path integrals at one-loop. These formulae are tested against lattice numerical data in the pure-scalar $O(4)$ model, where good agreement is found. Such formulae can be important tools for future works on confirming the triviality of the Higgs-Yukawa theory, or searching for alternative scenarios where strong-coupling fixed points exist.

\section{Acknowledgments}

DYJC and CJDL acknowledge the Taiwanese MoST grant number 105-2628-M-009-003-MY4. DYJC also thanks the financial support from Chen Cheng Foundation.

\section{References}

[1] A. Hasenfratz, K. Jansen, Y. Shen, Nucl. Phys. B394, 527 (1993), hep-lat/9207006

[2] P. Gerhold, K. Jansen, JHEP 09, 041 (2007), 0705. 2539

[3] P. Gerhold, K. Jansen, JHEP 10, 001 (2007), 0707. 3849

[4] J. Bulava, P. Gerhold, K. Jansen, J. Kallarackal, B. Knippschild, C.J.D. Lin, K.I. Nagai, A. Nagy, K. Ogawa, Adv. High Energy Phys. 2013, 875612 (2013), 1210. 1798

[5] C. Grojean, G. Servant, J.D. Wells, Phys. Rev. D71, 036001 (2005), hep-ph/0407019

[6] F.P. Huang, P.H. Gu, P.F. Yin, Z.H. Yu, X. Zhang, Phys. Rev. D93, 103515 (2016), 1511.03969

[7] P.H. Damgaard, A. Haarr, D. O'Connell, A. Tranberg, JHEP 02, 107 (2016), 1512 . 01963

[8] Q.H. Cao, F.P. Huang, K.P. Xie, X. Zhang (2017), 1708.04737

[9] J. de Vries, M. Postma, J. van de Vis, G. White (2017), 1710.04061

[10] M.S. Bilenky, A. Santamaria, Beyond the standard model with effective lagrangians, in 28th International Symposium on Particle Theory Wendisch-Rietz, Germany, August 30-September 3, 
1994 (1994), pp. 215-224, hep-ph/9503257, https: //inspirehep.net/record/382885/ files/arXiv:hep-ph_9503257.pdf

[11] D.Y.J. Chu, K. Jansen, B. Knippschild, C.J.D. Lin, A. Nagy, Phys. Lett. B744, 146 (2015), 1501.05440

[12] R. Fukuda, E. Kyriakopoulos, Nucl. Phys. B85, 354 (1975)

[13] L. O’Raifeartaigh, A. Wipf, H. Yoneyama, Nucl. Phys. B271, 653 (1986)

[14] O. Akerlund, P. de Forcrand, Phys. Rev. D93, 035015 (2016), 1508.07959

[15] E. Mølgaard, R. Shrock, Phys. Rev. D89, 105007 (2014), 1403. 3058

[16] H. Gies, M.M. Scherer, Eur. Phys. J. C66, 387 (2010), 0901.2459

[17] J. Frohlich, Nucl. Phys. B200, 281 (1982)

[18] M. Luscher, P. Weisz, Nucl. Phys. B318, 705 (1989)

[19] D.Y.J. Chu, K. Jansen, B. Knippschild, C.J.D. Lin, K.I. Nagai, A. Nagy, PoS LATTICE2015, 230 (2016), 1510.08620

[20] D.Y.J. Chu, K. Jansen, B. Knippschild, C.J.D. Lin, A. Nagy, PoS LATTICE2016, 217 (2016), 1611.00466

[21] E. Brezin, J. Zinn-Justin, Nucl. Phys. B257, 867 (1985)

[22] D.Y.J. Chu, K. Jansen, B. Knippschild, C.J.D. Lin, in preparation 